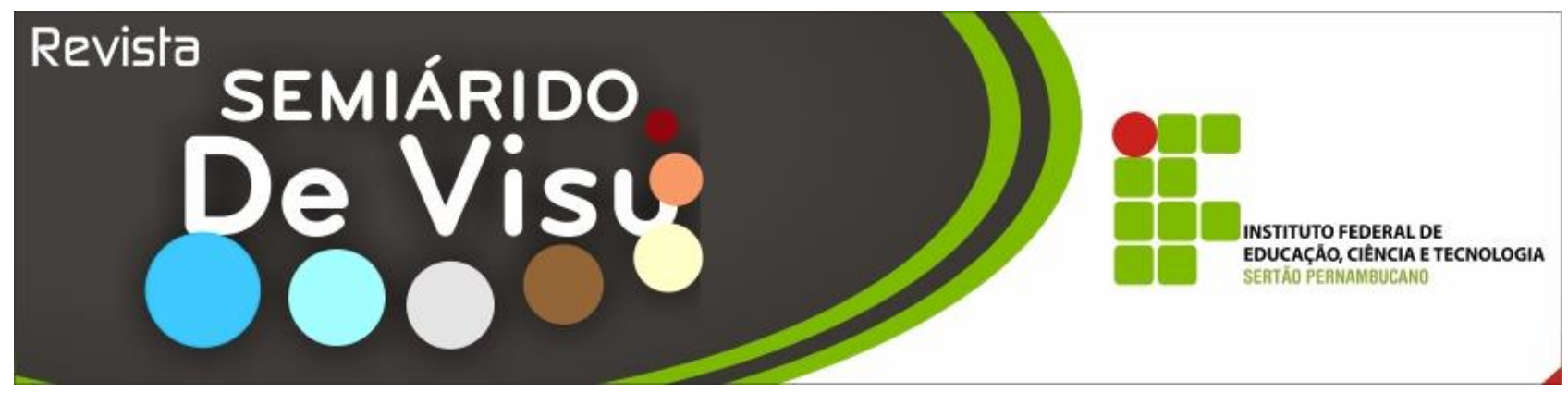

\title{
Dimetilformamida adicionada no sêmen de caprinos e seu efeito sobre a longevidade e funcionalidade da membrana espermática após criopreservação
}

\author{
Wildelfrancys Lima de Souza1, Elenice Andrade Morais ${ }^{2}$
}

\begin{abstract}
${ }^{1}$ Programa de Pós-Graduação em Ciências Veterinárias, Universidade Estadual do Ceará (UECE) - Campus Itaperi. Avenida Dr. Silas Muguba, 1700 - Fortaleza - Ceará - Brasil. CEP: 60740-000 / Telefone: (85) 3101-9601 / E-mail: wilde@ zootecnista.com.br ${ }^{2}$ Programa de Pós-Graduação em Ciência Animal, Universidade Federal do Vale do Sã Francisco (UNIVASF) - Campus Ciências Agrarias. Rodovia 407 Km 12, Lote 543, Projeto Nilo Coelho C1 - Petrolina - Pernambuco - Brasil. CEP: 563000-000 / Telefone:

(87) 2101-4810 / E-mail: elenice.moraes@univasf.edu.br
\end{abstract}

RESUMO: objetivou-se avaliar o efeito da dimetilformamida associada ou não ao glicerol no diluente de sêmen de caprinos antes da criopreservação sobre a longevidade e a funcionalidade da membrana espermática. Ejaculados de quatro caprinos foram coletados e submetidos aos seguintes tratamentos experimentas: Controle; Tris-gema (TG) $+2 \%$ de dimetilformamida (DMF2); TG + 3\% de DMF (DMF3); TG + 4\% de DMF (DMF4); TG + 5\% de DMF (DMF5); e $\mathrm{TG}+2 \%$ de glicerol $+2 \%$ DMF (DMF2G). Logo após resfriados a $5{ }^{\circ} \mathrm{C} / 2 \mathrm{~h}$, envasadas e colocadas sob vapor de nitrogênio por $7 \mathrm{~min}$, e então imersos no nitrogênio liquido e logo após, descongelados a $37{ }^{\circ} \mathrm{C} / 30$ segundos. As amostras foram analisadas quanto à longevidade e quanto ao teste hiposmótico. As variáveis foram avaliadas com ANOVA e teste de Tukey $(\mathrm{P}<0,05)$. A motilidade dos espermatozoides foi maior ao longo dos 120 min do pelo teste de termo resistência (TTR), nas amostras tratadas com DMF2, quando comparado às demais concentrações $(\mathrm{P}<0,05)$. Observou-se que os espermatozoides tratados com DMF2, apresentaram maior percentual de células com conservação na funcionalidade da membrana plasmática quando comparado aos demais tratamentos $(\mathrm{P}<0,05)$. Assim, o uso de $2 \%$ de dimetilformamida no diluente de sêmen caprino antes da criopreservação proporciona efeitos benéficos aos espermatozoides após descongelação, sendo indicado como substituto do glicerol na criopreservação de espermatozoides de caprinos.

Palavras-chave: espermatozoide, hiposmótico, membrana plasmática

\section{Dimethylformamide added in goat semen and its effect on the longevity and functionality of spermatic membrane after criopreservation}

\begin{abstract}
Aimed to evaluate the effect of dimethylformamide associated or not with glycerol in diluent of goat semen before cryopreservation on longevity and functionality of the plasma membrane. Ejaculated four goats were collected and subjected to the following treatments: Control; $2 \%$ of dimethylformamide (DMF2); 3\% of DMF (DMF3); $4 \%$ of DMF (DMF4); $5 \%$ of DMF (DMF5); and $2 \%$ of DMF plus $2 \%$ of glycerol (DMF2G). After cooling to $5{ }^{\circ} \mathrm{C} / 2 \mathrm{~h}$, packed and placed under nitrogen vapor for $7 \mathrm{~min}$, then immersed in liquid nitrogen and soon after, thawed at $37^{\circ} \mathrm{C} / 30$ seconds. Samples were analyzed for longevity and hyposmotic test. The variables were evaluated with ANOVA and Tukey's test $(\mathrm{P}<0.05)$. Sperm motility was higher over the $120 \mathrm{~min}$ of TTR in the samples treated with DMF2, when compared to the other concentrations $(\mathrm{P}<0.05)$. It was observed that spermatozoa treated with DMF2, presented higher percentage of cells with conservation in the functionality of the plasma membrane when compared to the other treatments $(\mathrm{P}<0.05)$. Thus, the use of $2 \%$ dimethylformamide in diluent of semen goat before cryopreservation it provides beneficial effects to spermatozoa after thawing, it is indicated as substitute of glycerol in cryopreservation of spermatozoa goats.
\end{abstract}

keywords: hyposmotic, plasma membrane, spermatozoa 


\section{Introdução}

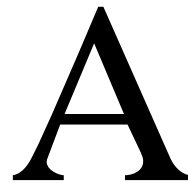

pós de 67 anos desde a primeira experiência bem-sucedida na congelação de espermatozoides (Smith \& Polge, 1950), os protocolos para a criopreservação de espermatozoides de caprino continuam a ser desenvolvidos devido à ampla gama de resultados encontrados para a motilidade espermática, considerada o parâmetro de escolha para determinar o grau de dano espermático, resultante durante o processo de criopreservação (Azeredo et al. 2001; Choe et al. 2006; Kathiravan et al. 2011; Kozdrowski et al. 2007). Estes valores percentuais variaram de $6 \%$ a $62 \%$, sendo as maiores porcentagens obtidas com a adição de antioxidantes ou colesterol nos diluentes (Sadia et al. 2008; Bucak et al. 2009; Bucak et al. 2010; Souza et al. 2016).

A melhoria na biotecnologia de criopreservação do sêmen caprino exige conhecimento aprofundado sobre as propriedades dos componentes utilizados nos diluentes (Sariozkan et al. 2010), assim, muitos crioprotetores permeáveis à membrana como o glicerol, dimetilsulfóxido, etilenoglicol e propilenoglicol, além de suas combinações, foram testados em espermatozoides (Singh et al. 1995; Kundu et al. 2000), sendo ainda, o crioprotetor penetrante mais utilizado, o glicerol. No entanto, o glicerol apresenta uma toxicidade aos espermatozoides (Holt 2000) e podendo induzir lesões osmóticas (Purdy 2006). Além do que, a adição do glicerol por si só pode causar certos danos estruturais e, portanto, reduzir a motilidade espermática (Salamon \& Maxwell 2000) podendo resultar em uma menor taxa de fertilidade quando a inseminação artificial é usado.

Nos garanhões (Alvarenga et al. 2005), coelhos (Okuda et al. 2007) e javalis (Bianchi et al. 2008), as amidas foram sugeridas como crioprotetores alternativos para a congelação espermática, principalmente para os indivíduos que eram mais sensíveis aos efeitos tóxicos do glicerol. Os efeitos crioprotetores das amidas são devidos ao seu peso molecular mais baixo $(73,09 \mathrm{~g} / \mathrm{mol})$ e a viscosidade em comparação com o glicerol $(92,05 \mathrm{~g} / \mathrm{mol})$, e pela sua maior permeabilidade à membrana, reduzindo assim a possibilidade de danos celulares causados pelo estresse osmótico (Ball \& Vo 2001; Dalimata \& Graham 1997). Além disso, a adição do radical metil (CH3) na molécula de amida aumenta a sua permeabilidade através da membrana das células espermáticas, melhorando assim a eficiência de na ação crioprotetora (Bianchi et al. 2008).

Assim, estudar o efeito da adição de dimetilformamida no diluente de sêmen caprino antes da criopreservação é uma alternativa ao glicerol e pode prover subsídios em novas técnicas para o transporte e armazenamento do sêmen. Portanto, objetivou-se avaliar o efeito da dimetilformamida associada ou não ao glicerol no diluente de sêmen de caprinos antes da criopreservação sobre a longevidade e a funcionalidade da membrana espermática.

\section{Material e métodos}

\section{Ética}

O presente estudo foi realizado após a aprovação institucional da Universidade Federal do Vale do São Francisco (Univasf), sob o protocolo $\mathrm{n}^{\mathrm{o}}$ 0005/161012, estando de acordo com os princípios éticos de experimentação animal do Comitê de Ética e Deontologia em Estudos e Pesquisas da Univasf.

\section{Local e período de execução}

O experimento foi realizado no setor de Caprinocultura e no Centro de Pesquisa em Suínos, Espécies Nativas e Silvestre (CPSENS), localizados no Campus de Ciências Agrárias da Univasf, situados no munícipio de Petrolina-PE (latitude 09 23'55" Sul e a uma longitude 40 $30^{\prime} 03^{\prime \prime}$ Oeste), estando a uma altitude de 376 metros, com precipitação média anual em torno de $300 \mathrm{~mm}$. A região apresenta temperatura média anual em torno dos $27^{\circ} \mathrm{C}$ e clima do tipo Bsh segundo a classificação de Köppen-Geiger (2007).

\section{Animais}


Foram utilizados quatro caprinos adultos, sendo dois da raça Boer e dois sem raça definida, com idade entre 2 a 4 anos, selecionados por meio de exame andrológico, sendo que todos apresentaram características espermáticas acima dos padrões mínimos, conforme estabelecido pelo Colégio Brasileiro de Reprodução Animal (CBRA 2013).

Os caprinos foram confinados em uma instalação contendo acesso a iluminação natural com dimensão de $2 \times 3 \mathrm{~m}$ de área coberta e com área de solário de $2 \times 4 \mathrm{~m}$, apresentando temperatura e umidade relativa média durante $\mathrm{o}$ período experimental de $28,7{ }^{\circ} \mathrm{C}$ e $45,9 \%$ respectivamente. Os caprinos foram mantidos isolados das fêmeas, recebendo água ad libitum e alimentação fornecida duas vezes ao dia, com dieta composta de capim elefante (Penissetum purpureum) picado, além de suplementação concentrada a base de farelo de milho, farelo de soja e mistura mineral. A relação volumoso:concentrado foi de 60:40 na forma de dieta total misturada, de acordo com as exigências para animais desta categoria segundo o National Research Council (NRC 2007).

\section{Coleta e processamento do sêmen}

Foram coletados 10 ejaculados de cada reprodutor caprino, por meio de vagina artificial para caprinos (Vargina $\operatorname{artificial}^{\circledR}$, Minitub, Berlim, Alemanha) totalizando 40 ejaculados. As coletas foram realizadas três vezes por semana para cada animal.

Após a coleta, o ejaculado foi transportado em uma caixa térmica para o CPSENS, mantido em banho-maria a $35{ }^{\circ} \mathrm{C}$, e avaliado quanto a alterações macroscópicas, como, presença de urina ou sangue. Foram utilizados apenas ejaculados que atendiam aos padrões considerados normais pelo CBRA (CBRA 2013).

Após avaliação, os ejaculados aprovados foram subdivididos em seis tubos de ensaio e diluídos em Tris e centrifugado a $2500 \mathrm{rpm}$, por 7 min. Após a centrifugação, o pellet foi diluído com $1 \mathrm{~mL}$ de Tris. Posteriormente, foram definidos os seguintes tratamentos experimentas: Controle, diluente a base de Trisgema $(\mathrm{TG})+2 \%$ de glicerol; $\mathrm{TG}+2 \%$ de dimetilformamida (DMF2); $\mathrm{TG}+3 \%$ de dimetilformamida (DMF3); $\mathrm{TG}+4 \%$ de dimetilformamida (DMF4); $\mathrm{TG}+5 \%$ de dimetilformamida (DMF5); e $\mathrm{TG}+2 \%$ de glicerol $+2 \%$ DMF $(\mathrm{DMF} 2 \mathrm{G})$, para concentração final de $120 \times 10^{6}$ espermatozoides $/ \mathrm{mL}$, determinado através da análise no fotômetro SDM6 (Minitub ${ }^{\circledR}$, Berlim, Alemanha), e mantidos em banho maria a 35 ${ }^{\circ} \mathrm{C}$.

Após distribuição dos seis tratamentos experimentais, em cada um dos 40 ejaculados, as amostras foram colocadas em Becker de 100 $\mathrm{mL}$ com água a $35{ }^{\circ} \mathrm{C}$, ficando acima do volume das amostras e então acondicionadas em câmara fria a $5{ }^{\circ} \mathrm{C}$ por duas horas. Depois, as amostras de cada tratamento foram envasadas em palhetas de $0,5 \mathrm{~mL}$ e lacradas com seladora (UltraSeal ${ }^{\circledR}$, Minitub, Berlim, Alemanha) e acondicionadas sob vapores do nitrogênio liquido, por 7 minutos, a $5 \mathrm{~cm}$ da lâmina líquida. Decorrido este tempo, as palhetas foram imersas no nitrogênio líquido ($196^{\circ} \mathrm{C}$ ) e estocadas em botijão criogênico para posterior análise.

\section{Descongelação do sêmen}

A descongelação foi realizada mergulhando-se as palhetas com as amostras de sêmen no descongelador automático $\left(\right.$ Cryofarm $^{\circledR}$, IMV, São Paulo, São Paulo, Brasil) por 30 segundos a $37^{\circ} \mathrm{C}$.

\section{Avaliação da motilidade espermática}

Duas palhetas de cada tratamento foram descongeladas conforme descrito acima para avaliação da motilidade espermática (progressiva), utilizando o sistema de análise computadorizada $\left(\mathrm{CASA}^{\circledR}\right.$, Minitub, Berlin, Alemanha), equipado com o SpermVision ${ }^{\circledR}$. Alíquotas de $8 \mu \mathrm{L}$ de cada amostra por tratamento foram analisadas, individualmente, sobre lâmina pré-aquecidas, a $37{ }^{\circ} \mathrm{C}$.

\section{Avaliação da longevidade dos espermatozoides descongelados}

A longevidade dos espermatozoides descongelados foi avaliada pelo teste de termo resistência (TTR), que consistiu no acondicionamento de 1,0 $\mathrm{mL}$ da amostra de 
sêmen em tubos plásticos de 1,5 mL em banhomaria a $37{ }^{\circ} \mathrm{C}$ por 120 minutos. Foram realizadas avaliações da motilidade total e motilidade progressiva no CASA, sendo as leituras realizadas nos tempos $0,30,60,90 \mathrm{e}$ 120 minutos de incubação.

\section{Funcionalidade da membrana plasmática}

Para avaliação da funcionalidade da membrana plasmática utilizou-se $\mathrm{o}$ teste hiposmótico (HOST) em câmara úmida, como descrito recentemente por Santos et al. (2015). Uma amostra de $100 \mu \mathrm{L}$ de sêmen descongelado diluído em $100 \mu \mathrm{L}$ da solução hiposmótica constituída por partes iguais de citrato tri-sódico e frutose obedecendo a concentração de $125 \mathrm{mOsm} / \mathrm{L}$, e incubados em banho maria durante $60 \mathrm{~min}$ a $37{ }^{\circ} \mathrm{C}$. Transcorrido esse tempo, as amostras foram formolizadas em solução de formol-salina tamponada na proporção de 10:1, estocadas em eppendorf e avaliado após sete dias.

Foram depositados $10 \mu \mathrm{L}$ da solução incubada sobre lâmina, coberta com lamínula para proceder à contagem de 200 espermatozoides por lâmina/tratamento,

\section{Resultados}

Os efeitos das diferentes concentrações de dimetilformamida sobre a motilidade espermática após diluição e resfriamento dos espermatozoides de caprinos estão apresentados na Tabela $1 \quad(\mathrm{P}<0,05) . \quad \mathrm{A}$ registrando-se a percentagem dos que apresentaram endosmose positiva (cauda enrolada), utilizando microscópio óptico com contraste de fase (DM 750 ${ }^{\circledR}$, Leica Microsystems, Heerbrugg, Suiça), em aumento de 400x.

O valor percentual de espermatozoides reativos ao HOST foi calculado utilizando a fórmula: $\operatorname{HOST}(\%)=(\%$ de alterações na região da cauda após HOST) - (\% de alterações na região da cauda antes do HOST).

\section{Análise Estatística}

O delineamento foi inteiramente casualizado em ensaio fatorial $4 \times 5$ (4 animais $\mathrm{x}$ 6 tratamentos). As análises dos parâmetros avaliados foram avaliadas utilizando-se o programa SAEG (UFV 1997). Todas as variáveis foram submetidas aos testes de normalidade (Teste de Lilliefors) e homocedacidade (Teste de Cochran e Bartlett), posteriormente as variáveis de distribuição normal foram submetidas à análise de variância e as médias comparadas pelo teste de Tukey com nível de significância de 5\%.

motilidade total e progressiva dos espermatozoides após as $2 \mathrm{~h}$ de resfriamento foi maior $(\mathrm{P}<0,05)$ nos espermatozoides que receberam o diluente DMF3 e DMF2G quando comparado ao controle e os diluentes DMF2, DMF4 e DMF5 (Figura 1).

Tabela 1 - Motilidade progressiva (média \pm D.P) dos espermatozoides descongelados de caprinos com diferentes concentrações de dimetilformamida ou associada ao glicerol

\begin{tabular}{ccc}
\hline & \multicolumn{2}{c}{ Motilidade progressiva (\%) } \\
\cline { 2 - 3 } Tratamentos & Diluído & Resfriado \\
\hline Controle & $89,0 \pm 1,77^{\mathrm{Aa}}$ & $87,1 \pm 2,21^{\mathrm{Aab}}$ \\
DMF2 & $89,4 \pm 1,65^{\mathrm{Aa}}$ & $87,7 \pm 1,07^{\mathrm{Aab}}$ \\
DMF3 & $90,1 \pm 1,83^{\mathrm{Aa}}$ & $89,4 \pm 0,64^{\mathrm{Aa}}$ \\
DMF4 & $90,0 \pm 1,47^{\mathrm{Aa}}$ & $88,0 \pm 1,52^{\mathrm{Aab}}$ \\
DMF5 & $89,5 \pm 1,52^{\mathrm{Aa}}$ & $85,7 \pm 1,16^{\mathrm{Bb}}$ \\
DMF2G & $90,6 \pm 0,79^{\mathrm{Aa}}$ & $89,4 \pm 1,02^{\mathrm{Aa}}$ \\
\hline
\end{tabular}

$\overline{\mathrm{A}, \mathrm{B}, \mathrm{C}}$ Médias seguidas de letras maiúsculas diferentes na mesma linha diferem entre si pelo teste de Tukey $(\mathrm{P}<0,05)$. Fonte: Pesquisa direta.

Os efeitos da dimetilformamida sobre a longevidade dos espermatozoides de caprinos estão apresentados na Tabela $2(\mathrm{P}<0,05)$. A motilidade progressiva dos espermatozoides foi maior ao longo dos $120 \mathrm{~min}$ do TTR, nas amostras tratadas com DMF2, quando 
comparado às demais concentrações testadas e

ao controle (Tabela 2; $\mathrm{P}<0,05$ ).

Tabela 2 - Longevidade dos espermatozoides descongelados de caprinos com diferentes concentrações de dimetilformamida ou associada ao glicerol durante o teste de termoresistência (TTR)

\begin{tabular}{lccccc}
\hline Tratamentos & \multicolumn{5}{c}{ TTR (minutos) } \\
\cline { 2 - 6 } & $\mathbf{0}$ & $\mathbf{3 0}$ & $\mathbf{6 0}$ & $\mathbf{9 0}$ & $\mathbf{1 2 0}$ \\
\hline Controle & $34,4 \pm 3,11^{\mathrm{B}}$ & $32,7 \pm 3,13^{\mathrm{B}}$ & $30,6 \pm 3,14^{\mathrm{B}}$ & $28,4 \pm 3,16^{\mathrm{B}}$ & $25,5 \pm 2,78^{\mathrm{C}}$ \\
DMF2 & $40,3 \pm 3,32^{\mathrm{A}}$ & $39,1 \pm 3,09^{\mathrm{A}}$ & $36,5 \pm 3,03^{\mathrm{A}}$ & $34,1 \pm 3,12^{\mathrm{A}}$ & $31,9 \pm 2,87^{\mathrm{A}}$ \\
DMF3 & $39,1 \pm 3,27^{\mathrm{A}}$ & $37,8 \pm 2,98^{\mathrm{AB}}$ & $34,3 \pm 2,74^{\mathrm{AB}}$ & $32,2 \pm 2,75^{\mathrm{AB}}$ & $27,8 \pm 3,06^{\mathrm{B}}$ \\
DMF4 & $37,5 \pm 2,85^{\mathrm{AB}}$ & $36,0 \pm 3,01^{\mathrm{AB}}$ & $33,7 \pm 3,00^{\mathrm{AB}}$ & $30,4 \pm 3,20^{\mathrm{AB}}$ & $27,4 \pm 2,85^{\mathrm{B}}$ \\
DMF5 & $36,6 \pm 3,19^{\mathrm{AB}}$ & $35,1 \pm 2,92^{\mathrm{AB}}$ & $32,9 \pm 2,90^{\mathrm{AB}}$ & $30,7 \pm 3,01^{\mathrm{AB}}$ & $26,1 \pm 3,12^{\mathrm{BC}}$ \\
DMF2G & $36,9 \pm 3,12^{\mathrm{AB}}$ & $36,2 \pm 3,21^{\mathrm{AB}}$ & $33,8 \pm 2,89^{\mathrm{AB}}$ & $30,9 \pm 3,10^{\mathrm{AB}}$ & $27,2 \pm 3,02^{\mathrm{B}}$ \\
\hline
\end{tabular}

${ }_{\mathrm{A}, \mathrm{B}, \mathrm{C}}$ Médias seguidas de letras diferentes na mesma coluna diferem entre si pelo teste de Tukey $(\mathrm{P}<0,05)$.

Os dados são expressos como média \pm D.P.

Fonte: Pesquisa direta.

Tabela 3 - Percentual dos espermatozoides descongelados de caprinos com diferentes concentrações de dimetilformamida ou associada ao glicerol reativos ao HOST

\begin{tabular}{cc}
\hline Tratamentos & HOST $(\%)$ \\
\hline Controle & $28,0 \pm 1,55^{\mathrm{B}}$ \\
DMF2 & $34,2 \pm 1,47^{\mathrm{A}}$ \\
DMF3 & $32,0 \pm 1,96^{\mathrm{AB}}$ \\
DMF4 & $30,3 \pm 1,54^{\mathrm{B}}$ \\
DMF5 & $29,3 \pm 1,63^{\mathrm{B}}$ \\
DMF2G & $28,9 \pm 1,79^{\mathrm{B}}$ \\
\hline
\end{tabular}

$\overline{\mathrm{A}, \mathrm{B}, \mathrm{C}}$ Médias seguidas de letras diferentes na mesma coluna diferem entre si pelo teste de Tukey $(\mathrm{P}<0,05)$.

Os dados são expressos como média \pm D.P.

Fonte: Pesquisa direta.

Os valores percentuais da funcionalidade da membrana plasmática dos espermatozoides de caprinos após o HOST (125mOsm) estão apresentados na Tabela 3. Observa-se que os espermatozoides de caprinos tratados com DMF2 antes da criopreservação, apresentaram maior percentual de células com conservação na funcionalidade da membrana plasmática quando comparado ao controle e demais tratamentos (Tabela 3; $\mathrm{P}<0,05$ ).

\section{Discussão}

Durante as etapas realizadas para a criopreservação do sêmen, as características de membrana dos espermatozoides são alteradas, 0 que interfere diretamente no seu potencial fecundante (Souza et al. 2016). Nessas etapas, ocorrem flutuações no volume celular, que contribuem para os danos na célula espermática, devido às alterações nos níveis hiperosmóticos (Purdy 2006). Dessa forma, com o intuito de minimizar os danos causados às células durante $\mathrm{o}$ processo de criopreservação, algumas substâncias vêm sendo estudadas e mostrando-se úteis como agentes crioprotetores (Bezerra et al. 2011).

Os crioprotetores mais utilizados nos diluentes seminais são as macromoléculas, como as proteínas da gema de ovo e o glicerol. Assim, esse estudo investigou o efeito de diferentes concentrações do crioprotetor dimetilformamida na longevidade e na funcionalidade da membrana espermática do espermatozoide de caprinos. Deste modo, a DMF foi utilizada para confirmar se, em baixas concentrações (2\%), sua ação protetora é maior ou igual a do glicerol e se, em concentrações elevadas (5\%), é tóxico aos espermatozoides.

Assim, após o resfriamento a $5{ }^{\circ} \mathrm{C}$, a adição de crioprotetores no sêmen caprino proporcionou um maior percentual de motilidade progressiva com o uso de DMF. 
Estes resultados são semelhantes aos encontrados no sêmen canino (Lopes et al. 2009). Sabe-se que a adição de um crioprotetor a uma suspensão pode afetar sua condutividade hidráulica e interferir com o estresse osmótico, os quais as células são expostas durante a curva de congelação, nas etapas de resfriamento e congelação (Gilmore et al. 1995). Onde, com a redução da temperatura, a pressão osmótica assistida por adição de DMF é menos deletéria para as células espermáticas do que o causado pelo glicerol (Lopes et al. 2009).

Os resultados de motilidade espermática após a descongelação demonstraram que os padrões percentuais avaliados por CASA (motilidade progressiva), foram mais bem preservados no uso de DMF do que com o glicerol. Estes resultados foram semelhantes aos relatados para o touro (Gonzalez 2004) e em espermatozoides do cão (Lopes et al. 2009). Nesta última espécie, foi levantada a hipótese de que as diferenças na susceptibilidade espermática aos crioprotetores podem afetar a adaptação de substâncias para várias espécies, talvez devido às condições tóxicas desconhecidas. Sugeriu-se também que as diferenças entre espécies na quantidade e no tipo dos fosfolipídios, podem interferir com a estabilidade da membrana do espermatozoide durante a criopreservação (Hammersted et al. 1990).

Os resultados desse estudo demonstraram que a adição de $2 \%$ de DMF como crioprotetor, possibilitou uma maior porcentagem de motilidade progressiva nos espermatozoides durante os 120 minutos do TTR, enquanto que a adição de concentrações acima de $2 \%$ de DMF não apresentou resultado similar. Além do que, o valor percentual de motilidade progressiva observada na concentração de DMF a 2\% no tempo de 120 min, foi bastante superior ao valor percentual observado no tratamento controle, o qual utiliza o glicerol como crioprotetor. Os resultados acima descritos podem ser justificados pelo fato que, a redução na motilidade espermática observada nas amostras com concentrações acima de 2\% de DMF está associada aos efeitos tóxicos exercidos sobre a célula espermática, ao elevar as concentrações de DMF. Todavia, a
DMF mesmo na concentração de $2 \%$, quando associado ao glicerol, provavelmente, potencializou seu efeito tóxico, assim, a toxicidade à célula espermática foi aumentada, fato responsável pelas lesões evidenciadas quanto à funcionalidade da membrana plasmática.

Sabe-se que a motilidade espermática é um fator importante no potencial fecundante de um reprodutor por importância na migração no trato genital e na interação do gameta para fecundação (Suarez \& Pacey, 2006). A DMF ou N-metilformamida, um produto de biotransformação da DMF, está associada a efeitos adversos sobre as mitocôndrias dos espermatozoides (Chang et al. 2004), mas como isso acontece não está claro. Sabe-se que a função mitocondrial é um dos fatores etiológicos que são reconhecidos para a redução da motilidade espermática. A eficácia propulsiva do espermatozoide é primariamente dependente da função mitocondrial. As mitocôndrias localizadas na peça intermediária dos espermatozoides fornecem a energia para geração e propagação da onda flagelar (Perumal et al. 2013).

Com base nessas informações, pode-se sugerir que devido a maior atividade mitocondrial observada na concentração de DMF a $2 \%$, a produção e os níveis de ATP intracelular são mais elevados, sendo atribuída a minimização do efeito tóxico exercida pela baixa concentração do crioprotetor nas mitocôndrias espermáticas contra as injurias que podem prejudicar a produção de ATP, bem como as estruturas flagelares. Nos espermatozoides, a produção de ATP supre várias atividades celulares e eventos bioquímicos necessários para que a fecundação seja bem-sucedida, tais como a capacitação, reação acrossômica e a motilidade espermática (Miki 2007). Dessa forma, a maior disponibilidade de ATP contribuiu para uma maior motilidade espermática, observada após a criopreservação na concentração de DMF a $2 \%$, em comparação com os demais grupos experimentais.

Os resultados do teste hiposmótico demonstraram que o percentual de dobramento de cauda pode estar relacionado aos danos que a cauda espermática sofre ao longo das etapas 
para a criopreservação, o que reduz a reação dos espermatozoides ao aumento do volume celular, devido à baixa osmolaridade. Além disso, o próprio meio de congelação pode induzir ao estresse osmótico, o que resultaria em lesões na estrutura celular (Correa \& Zavos, 1994). Além disso, a presença da proteína de canal de água, Aquaporina 7 (AQP7), na membrana plasmática, que tem como função o transporte de glicerol (Curry 2000), tenha favorecido a maior penetração dessa substância no interior das células. Portanto, é possível que, neste estudo, a redução da motilidade progressiva e, funcionalidade da membrana plasmática ocasionadas especialmente pelo glicerol, tenha sido determinada pela ocorrência de distúrbios osmóticos, os quais comprometem a integridade dos espermatozoides (Snoeck et al., 2007).

Os resultados sugerem que a adição de $2 \%$ de DMF exerce melhor efeito crioprotetor sobre a membrana plasmática durante a congelação-descongelação, favorecendo a redução do efeito deletério do estresse osmótico durante a criopreservação. Tal sugestão é reforçada pelo fato que, modificações nos diluidores seminais podem determinar distúrbios osmóticos (Juliani \& Henry, 2008), em que, tanto as altas osmolaridades, como as baixas, são prejudiciais à sobrevivência dos espermatozoides, como consequência de alterações em suas estruturas e funções (Luzardo et al., 2010). Além do que, em decorrência de sua composição, a membrana plasmática dos espermatozoides possui elevada permeabilidade ao glicerol (Salamon \& Maxwell, 2000), o que determina a ação tóxica desse crioprotetor sobre as células (Alvarenga et al., 2005).

Sabe-se que não só a natureza, mas também a concentração de crioprotetores podem interferir nos resultados pósdescongelação (Holt 2000). Além disso, Bezerra et al. (2011) demonstraram recentemente que o sêmen caprino criopreservado utilizando 6\% de DMF resultou em uma taxa de prenhez de 27,3\%. Estes resultados indicam que a DMF tem um potencial como crioprotetor para o sémen caprino, mas outras concentrações desta substância, e também outros protocolos de congelação devem ser testados. Assim, a DMF em concentrações até $2 \%$ poderia ser testada como um crioprotetor alternativo para o sêmen caprino, cujo sêmen apresenta uma baixa qualidade pós descongelação, quando congelado com o glicerol, uma vez que este efeito positivo foi previamente comprovado para garanhão (Alvarenga et al. 2005).

\section{Conclusões}

Em conclusão, os resultados demonstraram a possibilidade de se utilizar a DMF como um crioprotetor alternativo para o congelamento do sémen caprino na concentração de $2 \%$. Proporcionando efeitos benéficos aos espermatozoides após descongelação, apresentando maior capacidade de preservar a qualidade espermática, sendo indicado como substituto do crioprotetor glicerol na criopreservação de espermatozoides de caprinos.

\section{Agradecimentos}

Ao Centro de Pesquisa em Suínos, Espécies Nativas e Silvestre (CPSENS) do CPGCA/UNIVASF por toda infraestrutura (APQ-1072-5.04/12 e APQ-0227-5.04/10) e suporte para a realização da pesquisa. A Pró Reitoria de Pesquisa e Pós-Graduação da UNIVASF pelo suporte financeiro para aquisição de equipamentos (Projeto financiado em Edital 17/2013).

\section{Referências}

ALVARENGA, M.A.; PAPA, F.O.; LANDIMALVARENGA, F.C.; MEDEIROS, A.S. Amides as cryoprotectants for freezing stallion semen: a review. Anim. Reprod. Sci. 89(14):105-113, 2005.

AZEREDO, Z.A.; ESPER, C.R. \& RESENDE, K.T. Evaluation of plasma membrane integrity of frozen-thawed goat spermatozoa with or without seminal plasma, Small Rumin. Res. 41:257-263, 2001. 
BALL, B.A. \& VO, A. Osmotic tolerance of equine spermatozoa and the effects of soluble cryoprotectants on equine sperm motility, viability and mitochondrial membrane potential, J. Androl. 22:1061-1069, 2001.

BEZERRA, F.S.B.; CASTELO, T.S.; ALVES, H.M.; OLIVEIRA， I.R.S.; LIMA， G.L.; PEIXOTO, G.C.X.; BEZERRA, A.C.S.D.; SILVA, A.R. Objective assessment of the cryoprotective effects of dimethylformamide for freezing goat sêmen. Cryobiology, 63:263266, 2011.

BIANCHI, I.; CALDERAM, K.; MASCHIO, E.F.; MADEIRA, E.M.; ULGUIM, R.R.; CORCINI, C.D. Evaluation of amides and centrifugation temperature in boar semen cryopreservation. Theriogenology. 69(5):632638, 2008.

BUCAK, M.N.; SARIOZKAN, S.; TUNCER, P.B.; ULUTAS, P.A.; AKCADAG, H.I. Effect of antioxidants on microscopic semen parameters, lipid peroxidation and antioxidant activities in Angora goat semen following cryopreservation, Small Rumin. Res. 81: 9095, 2009.

BUCAK, M.N.; SARIOZKAN, S.; TUNCER, P.B.; SAKIN, F.; ATESSAHIN, A.; KULAKSIZ, R.; CEVIK, M. The effect of antioxidants on post-thawed Angora goat (Capra hircus ancryrensis) sperm parameters, lipid peroxidation and antioxidant activities, Small Rumin. Res. 89:24-30, 2010.

CHANG, H.Y.; SHIH, T.S.; GUO, Y.L.; TSAI, C.Y.; HSU, P.C. Sperm function in workers exposed to $\mathrm{N}, \mathrm{N}$-dimethylformamide in the synthetic leather industry, Fertil. Steril. 81:1589-1594, 2004.

CHOE, C.Y.; KIM, J.G.; CHO, S.R.; SON, D.S.; KIM, Y.K.; BALASUBRAMANIAN, S.; CHOE, S.Y.; RHO, G.J. Influence of seasons, extenders, slow and rapid freezing on seminal characters in Korean native bucks, Reprod. Domest. Anim. 41:55-60, 2006.
Colégio Brasileiro de Reprodução AnimalCBRA. Manual para exame andrológico e avaliação de sêmen animal. 3.ed. Belo Horizonte. 104 p, 2013.

CORREA, J.R. \& ZAVOS, P.M. The hypoosmotic swelling test: its employment as an assay to evaluate the functional integrity of the frozen-thawed bovine sperm membrane. Theriogenology. 42(2):351-360, 1994.

CURRY, M.R. Cryopreservation of semen from domestic livestock. Rev. Reprod. 5(1):46-52, 2000 .

DALIMATA, A.M. \& GRAHAM, J,K. Cryopreservation of rabbit spermatozoa using acetamide in combination with trehalose and methylcellulose, Theriogenology 49:831-841, 1997.

GILMORE, J.A.; MCGANN, L.E.; LIU, J.; GAO, D.Y.; PETER, A.T.; KLEINHANS, F.W.; CRITSER, J.K. Effect of cryoprotectant solutes on water permeability of human spermatozoa, Biol. Reprod. 53:985-995, 1995.

GONZALEZ, R.A.R. Effect of cryopreservaton using different freezing techniques and cryoprotectants on sperm parameters and membrane integrity of bull sperm, Thesis, University of Sao Paulo, Pirassununga, Brazil, 2004.

HAMMERSTED, R.H.; GRAHAM, J.K. \& NOLAN, J.P. Cryopreservation of mammalian sperm: what we ask them to survive, J. Androl. 11:73-88, 1990.

HOLT, W.V. Basic aspects of frozen storage of semen, Anim. Reprod. Sci. 62:3-22, 2000.

JULIANI, G.C. \& HENRY, M. Efeito do glicerol, etilenoglicol, acetamida e leite desnatado na criopreservação de espermatozoides equinos. Arq Bras Med Vet Zootec. 60(5):1103-1109, 2008.

KATHIRAVAN， P.; KALATHARAN， J.; KARTHIKEYA， G.; RENGARAJAN， K.; 
KADIRVEL, G. Objective sperm motion analysis to assess dairy bull fertility using computer aided system - a review, Reprod. Domest. Anim. 46:165-172, 2011.

KÖPPEN-GEIGERPEEL, M.C.; FINLAYSON, B.L. \& MCMAHON, T.A. Updated world map of the Köppen-Geiger climate classification. Hydrol. Earth Syst. Sci. 11:1633-1644, 2007.

KOZDROWSKI, R.; DUBIEL, A.; BIELAS, W.; DZIECIOE, M. Two protocols of cryopreservation of goat semen with the use of computer-assisted sêmen analysis system, Acta Vet. Brno. 76:601-604, 2007.

KUNDU, C.N.; CHAKRABORTY, J.; DUTTA, P.; BHATTACHARYYA, D.; GHOSH, A.; MAJUMDER, G.C. Development of a simple sperm cryopreservation model using a chemically defined medium and goat cauda epididymal spermatozoa, Cryobiology 40:117125, 2000.

LOPES, K.R.F.; COSTA, L.L.M.; LIMA, G.L.; SOUZA, A.L.P.; SILVA, A.R. Dimethylformamide is no better than glycerol for cryopreservation of canine semen, Theriogenology 72:650-654, 2009.

LUZARDO, B.; CASTRO, M.C.; GAMBOA, F.A.; LOPEZ, M.A.; LOPEZ, A.Y.R.A. Osmolarity of coconut water (Cocos nucifera) based diluents and their effect over viability of frozen boar semen. Am J Anim Vet Sci. 5(3):187-191, 2010.

MIKI, K. Energy metabolism and sperm function. Soc. Reprod. Fertil. (Suppl.) 65:309-325, 2007.

National Requirement Council - NRC. Nutrient Requirements of Small Ruminants: Sheep, Goats, Cervids, and New World Camelids. National Academy of Science, Washintgton, D.C. 347p, 2007.

OKUDA, Y.; SEITA, Y.; HISAMATSU, S.; SONOKI, S.; SHINO, M.; MASAOKA, T.; INOMATA, T.; KAMIJO, S.I.;
KASHIWAZAKI, N. Fertility of spermatozoa cryopreserved with $2 \%$ acetamide or glycerol through artificial insemination in the Japanese White rabbit, Exp. Anim. 56:29-34, 2007.

PERUMAL, P.; VUPRU, K. \& RAJKHOWA, C. Effect of addition of taurine on the liquid storage $\left(5^{\circ} \mathrm{C}\right)$ of mithun (Bos frontalis) semen. Vet. Med. Int. 2013(4):1-7, 2013.

PURDY, P.H. A review on goat sperm cryopreservation, Small Rumin. Res. 63:215225, 2006.

SADIA, A.; RASHEDUL, I.M.; YAHIA, K.M.A.M.; SHARMIN, A.Q. Cryopreservation of Black Bengal buck semen: effects of diluents and freezing on sperm motility and morphology, Anim. Sci. J. 79:550-553, 2008.

SALAMON, S. \& MAXWELL, W.M.C. Storage of ram semen. Anim Reprod Sci. 62(1-3):77-111, 2000.

SANTOS, M.A.M.; GRADELA, A.; MORAES, E.A.; SOUZA, W.L.; ALVES, N.G.; COSTA, J.M.S.; MATOS, W.C.G. Características do sêmen a fresco e descongelado de garanhões da raça Nordestina. Pesqui. Vet. Bras. 35(11):925-932, 2015.

SARIOZKAN, S.; BUCAK, M.N.; TUNCER, P.B.; TASDEMIR, U.; KINET, H.; ULUTAS, P.A. Effects of different extenders and centrifugation/washing on postthaw microscopicoxidative stress parameters and fertilizing ability of Angora buck sperm, Theriogenology 73:316-323, 2010.

SINGH, M.P.; SINHA, A.K. \& SINGH, B.K. Effect of cryoprotectants on certain seminal attributes and on the fertility of buck spermatozoa, Theriogenology 43:1047-1053, 1995.

SMITH, A.H. \& POLGE, C. Survival of spermatozoa at low temperatures, Nature 166:668-671, 1950. 
SNOECK, P.P.N.; HENRY, M. \& MELO, M.I.M. EFEITO DE DIFERENTES DILUIDORES SOBRE A VIABILIDADE espermática pós-descongelação de sêmen eqüino. Arquivo Brasileiro de Medicina Veterinária e Zootecnia, Belo Horizonte, v.59, n.1, p.56-64, 2007.

SOUZA, W.L.; MORAES, E.A.; COSTA, J.M.S.; GRAHAM, J.K. Cholesterol-loaded cyclodextrin in fresh goat sperm improves cryosurvival rates. Rev. Bras. Ciênc. Vet. 23(12):93-98, 2016.

SUAREZ, S.S. \& PACEY, A.A. Sperm transport in the female reproductive tract, Hum. Reprod. Update 12:23-37, 2006.

Universidade Federal de Viçosa (UFV)-SAEG. Manual de utilização do programa SAEG (Sistema para Análise Estatística e Genéticas). Viçosa: UFV. p149, 1997. 